\title{
Optogenetic Localization and Genetic Perturbation of Saccade-Generating Neurons in Zebrafish
}

\author{
Peter J. Schoonheim, ${ }^{\star}$ Aristides B. Arrenberg, ${ }^{\star}$ Filippo Del Bene, and Herwig Baier \\ Department of Physiology, Programs in Neuroscience, Genetics and Developmental Biology, University of California, San Francisco, San Francisco, \\ California 94158-2324
}

The optokinetic response (OKR) to a visual stimulus moving at constant velocity consists of a series of two alternating components, a slow phase, during which the eyes follow the stimulus, and a quick phase, which resets the eyes to begin a new response cycle. The quick phases of the OKR resemble the saccades observed during free viewing. It is unclear to what extent the premotor circuitry underlying these two types of jerky, conjugate eye movements is conserved among vertebrates. Zebrafish (Danio rerio) larvae, broadly expressing halorhodopsin (NpHR) or channelrhodopsin-2 (ChR2) in most neurons, were used to map the location of neurons involved in this behavior. By blocking activity in localized groups of NpHR-expressing neurons with an optic fiber positioned above the head of the fish and by systematically varying the site of photostimulation, we discovered that activity in a small hindbrain area in rhombomere 5 was necessary for saccades to occur. Unilateral block of activity at this site affected behavior in a direction-specific manner. Inhibition of the right side suppressed rightward saccades of both eyes, while leaving leftward saccades unaffected, and vice versa. Photostimulation of this area in ChR2-transgenic fish was sufficient to trigger saccades that were precisely locked to the light pulses. These extra saccades could be induced both during free viewing and during the $\mathrm{OKR}$, and were distinct in their kinetics from eye movements elicited by stimulating the abducens motor neurons. Zebrafish double indemnity (didy) mutants were identified in a chemical mutagenesis screen based on a defect in sustaining saccades during OKR. Positional cloning, molecular analysis, and electrophysiology revealed that the didy mutation disrupts the voltage-gated sodium channel Scn1lab (Nav1.lb). ChR2 photostimulation of the putative hindbrain saccade generator was able to fully reconstitute saccades in the didy mutant. Our studies demonstrate that an optogenetic approach is useful for targeted loss-offunction and gain-of-function manipulations of neural circuitry underlying eye movements in zebrafish and that the saccade-generating circuit in this species shares many of its properties with that in mammals.

\section{Introduction}

Quick eye movements, or saccades, assist vision by redirecting the gaze and thus expanding, or adjusting, the field of view. The neural mechanisms responsible for eye position and saccade initiation have been studied extensively in mammals (Fuchs and Kaneko, 1981; Scudder et al., 2002; Glimcher, 2003). Here, the nucleus prepositus (NPH) and the vestibular nucleus in the brainstem are important for horizontal eye movements and contain neurons with eye position and velocity signals (LopezBarneo et al., 1982; Tomlinson and Robinson, 1984). Horizontal saccades are generated by a neural circuit called the burst gener-

Received 0ct. 19, 2009; revised March 22, 2010; accepted March 25, 2010.

This work was supported by National Institutes of Health (NIH) Grant R01 NS053358, a Sandler Opportunity Award, the Byers Award for Basic Science, and the NIH Nanomedicine Development Center "Optical Control of Biological Functions" (to H.B.). A.B.A. was funded by a stipend from the Boehringer Ingelheim Foundation and a Julius R. and Patricia A. Krevans Fellowship. F.D.B. was supported by a Human Frontier Science Program Postdoctoral Fellowship and a Sandler Postdoctoral Award. We thank A. Goldin, A. Ribera, and K. Deisseroth for providing the rat Nav1.2 plasmid, the zebrafish Scn1lab morpholino, and the NpHR and ChR2 constructs, respectively. T.R. Thiele generated the construct for the $\operatorname{Ig}($ UAS:ChR2(H134R)-mCherry) transgenic line. The Xenopus 0ocyte two-electrode voltage-clamp recordings were performed in L.Y. Jan's laboratory with the help of T. Jin. J. Huisken assisted with the laser setup.

*P.J.S. and A.B.A. contributed equally to this article.

Correspondence should be addressed to Herwig Baier, Department of Physiology, Rock Hall, 1550 4th Street, San Francisco, CA 94158-2324. E-mail: herwig.baier@ucsf.edu.

DOI:10.1523/JNEUROSCI.5193-09.2010

Copyright $\odot 2010$ the authors $\quad 0270-6474 / 10 / 307111-10 \$ 15.00 / 0$ ator, which consists of excitatory burst neurons (EBNs) in the paramedian pontine reticular formation, inhibitory burst neurons (IBNs), and omnipause neurons (OPNs) (Strassman et al., 1986a,b). Preceding a saccade, the high constant firing rate of OPNs is inhibited by glycinergic inputs (Kanda et al., 2007). This pause of OPN inhibition gives rise to a period of short, rapid firing of both EBNs and IBNs (Scudder et al., 2002). Both types of burst neurons directly synapse with the abducens motor neurons (Yoshida et al., 1982). The EBNs project to the ipsilateral abducens nucleus to excite agonist motor neurons (Strassman et al., 1986a), whereas the IBNs inhibit antagonist motor neurons in the contralateral abducens nucleus (Strassman et al., 1986b). Together, this activation pattern results in a quick, synchronous (conjugate) movement of both eyes in the same direction.

The task of voluntary saccades during free viewing in primates and cats is to bring the image of an eccentric visual target into the fovea. In contrast, the eyes of commonly studied teleost fish do not possess a fovea. However, these animals exhibit spontaneous "scanning" saccades during free viewing. Little is known about the teleost hindbrain circuitry for saccade generation. In adult goldfish, brain regions that control eye movements were named area I and area II (Pastor et al., 1994; Aksay et al., 2000, 2001, 2007). It was shown that area I predominantly sends projections to the abducens nucleus (Aksay et al., 2000, 2001) in contrast to area II, which is exclusively precerebellar (Beck et al., 2006; Straka 
et al., 2006). Inactivation of area I neurons using lidocaine results in a dramatic eye drift and velocity bias but does not inhibit saccade initiation. In contrast, when lidocaine is injected between area I and area II, saccades are no longer generated, consistent with the existence of a burst generator at this position (Pastor et al., 1994). Although area I and II combined carry out a similar function to the NPH in primates and cats, their homology to mammalian nuclei is uncertain (Beck et al., 2006).

Both mammals and teleosts exhibit another type of jerky, conjugate eye movement as part of their vestibulo-ocular reflex (Marsh and Baker, 1997). These reflex movements resemble saccades in speed and amplitude and punctuate periods during which the visual image is stabilized on the retina. Such "resetting saccades" can be elicited by visual stimulation alone, resulting in the well known optokinetic response (OKR). A drifting grating, composed of black and white stripes, that rotates around an agarose-embedded zebrafish larva at constant speed, evokes a series of highly regular eye-following movements, which counteract the perceived retinal slip (Easter and Nicola, 1996; Beck et al., 2004). In regular intervals, as the eyes move to an extreme viewing angle, saccade-like movements then reset their orientation, and the cycle begins anew.

Despite a long-standing interest in the zebrafish OKR as a sensitive readout of visual function (Brockerhoff et al., 1995; Neuhauss et al., 1999; Muto et al., 2005), little is known about the underlying neural pathway. While the pathways generating eye movements converge on a shared set of oculomotor neurons in all vertebrates, it is not known whether different types of saccades are produced by the same premotor circuits and whether teleosts possess a region that is homologous to the mammalian burst generator. To gain insight into this issue, we have chosen here a functional approach in zebrafish (Arrenberg et al., 2009). We generated transgenic zebrafish larvae expressing halorhodopsin (NpHR) in the entire CNS. Small areas of the brain were then photostimulated with a thin optic fiber, while the animal's OKR to a drifting grating was monitored with a video camera. $\mathrm{NpHR}$ stimulation blocked saccades at only one position in the hindbrain. This region shares several characteristics with the burst generator of mammals. Activation of the identical region with channelrhodopsin-2 (ChR2) evoked conjugate saccades in wildtype larvae. The same treatment also restored saccades in double indemnity (didy) mutants, which fail to sustain saccades during long exposure to a moving grating due to mutation of a voltagegated sodium channel. These experiments help to localize an important brain function in zebrafish larvae, invite further work on homologies between teleost and mammalian hindbrain circuits, and show that optogenetic intervention can be successfully used to reverse a neurological defect in an animal model.

\section{Materials and Methods}

Fish maintenance and genotypes of wildtype strains, mutants, and transgenics. Zebrafish (Danio rerio) of the standard laboratory strains TL (Tüpfel long fin), TLN (melanin-deficient TL fish that carry mitfa/na$\mathrm{cre}^{\text {s170 }}$ and mitfa/nacre ${ }^{\text {s184 }}$ mutations), and WIK were maintained at $28.5^{\circ} \mathrm{C}$ on a $14 \mathrm{~h} \mathrm{light} / 10 \mathrm{~h}$ dark cycle. For behavioral experiments, we used a mix of TL and TLN. For genetic mapping of the mutation, we obtained a hybrid between the TL and WIK strains. The Committee on Animal Research of the University of California, San Francisco, approved all experiments.

Two alleles of the didy mutant, $d i d y^{5552}$ and $d i d y^{5390}$, were discovered in a chemical mutagenesis screen using the OKR as a screening assay (Muto et al., 2005). Transgenic lines Et(E1b:Gal4-VP16)s1101t, Et(hsp(600bp):Gal4VP16)s1020t, $\mathrm{Tg}$ (UAS:Kaede)s1999t, Tg(UAS:NpHR-mCherry)s1989t, $\mathrm{Tg}(U A S: N p H R-e Y F P) s 1987 t$, and $\operatorname{Tg}(U A S: C h R 2(H 134 R)-e Y F P) s 1990 t$ were previously described (Scott et al., 2007; Arrenberg et al., 2009). $\operatorname{Tg}$ (UAS: ChR2(H134R)-mCherry)s1986t was newly generated for this project by established procedures. Line and allele designations follow zebrafish nomenclature conventions (http://zfin.org/).

Optokinetic response assay. A miniature surround movie theater was built for visual stimulation. Four $72 \mathrm{~mm}$ LCD (liquid crystal display) monitors (Aeicomp) were arranged in a square and driven by one TripleHead2Go box (Matrox) and a dual-head GPU. A moving pattern of stripes $\left(10^{\circ} / \mathrm{s}, 18 \mathrm{cycles} / 360^{\circ}\right.$, maximal contrast) was generated in Matlab (MathWorks) with the Psychtoolbox suite (www.psychtoolbox.org) (Brainard, 1997; Pelli, 1997). The pattern was warped in such a way that the temporal frequency and angular velocity were constant around the virtual circular perimeter of the movie theater. Larvae [ $5 \mathrm{~d}$ postfertilization (dpf)] were mounted in either $1.5 \%$ low-melting agarose or $2.5 \%$ methylcellulose, the agarose surrounding the eyes was removed, and larvae were placed on a stage in the movie theater. A white, high-power LED (light-emitting diode) was used as backlight for the CCD video camera. The frame rate was 15 frames/s for saccade velocity measurements and 2 frames/s for all other experiments. Spatial frequency, contrast, and time of reversal of the sine-wave gratings were adjusted using a custom-made LabVIEW program (National Instruments). The velocity of the eyes was calculated by performing linear regression of the eye movement between two eye reversals (either a saccade or stimulusinduced directional change of the eye). To calculate the gain of the OKR slow phase, the velocity of the eyes was divided by the velocity of the stimulus.

Manipulation of neural activity with NpHR and ChR2. Zebrafish carrying $\mathrm{Tg}$ (UAS:ChR2(H134R)-eYFP)s1990t, Tg(UAS:ChR2(H134R)$m$ Cherry)s1986t, or $\operatorname{Tg}(U A S: N p H R$-mCherry)s1989t were mated to zebrafish carrying Et(E1b:Gal4-VP16)s1101t. For adult carriers of the TLN/TL hybrid strain, 25\% of the larvae were melanophore deficient (homozygous nacre). Light stimulation and simultaneous behavioral analysis of zebrafish ( $5 \mathrm{dpf}$ ) larvae were performed as described previously (Arrenberg et al., 2009). Briefly, the multimode, low numerical aperture $50 \mu \mathrm{m}$ fiber (AFS50/125Y; Thorlabs) was coupled to the singlemode fiber of a laser source (UV, $365 \mathrm{~nm}$; argon, $488 \mathrm{~nm}$; krypton, 568 $\mathrm{nm}$; HeNe, $633 \mathrm{~nm}$ ). Placement of the fiber was performed using a micromanipulator. For some animals, stimulation of the hindbrain did not evoke saccades, and these animals were excluded from analysis. The $\mathrm{Tg}$ (UAS:ChR2(H134R)-eYFP)s1990t line exhibits random variegation, which may result in diminished ChR2 expression in the putative saccade generator in a subset of the animals.

Positional cloning and genotyping. To identify the didy gene, we used microsatellite mapping as described before (Shimoda et al., 1999). Initially, genomic DNA was isolated from 24 pooled mutant or wildtype sibling larvae. Subsequently, linked markers were used to determine the recombination frequencies, and thus the putative distances to the mutation, in DNA isolated from 600 individual larvae. Mutant larvae were selected by their black appearance, which is indicative of a neuroendocrine deficit in controlling pigment aggregation. The markers $z 6601-6$ and $z 6601-8$ were identified by interrogation of the genomic sequence for simple sequence repeats (SSRs). Candidate SSRs were empirically tested for whether they were polymorphic between the WIK and TL strains. Oligonucleotides for these new markers are: $z 6601-6 F\left(5^{\prime}\right.$-gcc aga gtt aag ggt ttc aaa gca c- $\left.3^{\prime}\right), z 6601-6 R v\left(5^{\prime}\right.$-gag ttg ggc tgt ggt cag gtt tc- $\left.3^{\prime}\right)$, $z 6601-8 F w$ ( $5^{\prime}$ tag atg tgg gga cat ttg gtc tcc- $\left.3^{\prime}\right)$, and $z 6601-8 R v$ ( $5^{\prime}$-agt ctt ttc tca tgt gcc tgt ttg $\left.\mathrm{c}-3^{\prime}\right)$.

Genotyping of didy mutants was performed by means of a restriction fragment length polymorphism marker. The $d i d y^{5552}$ mutation introduces a novel AcuI restriction site into scn1lab. Digestion of a PCR fragment spanning the $d i d y^{552}$ mutation with AcuI results in two smaller fragments in fish carrying the $d i d y^{552}$ allele.

Morpholino phenocopy and in situ RNA hybridization. Phenocopy of the didy $y^{552}$ loss-of-function mutation was performed by morpholinomediated knock down of scn1lab. A morpholino targeted to the atg translation start site of scn1lab (5'-agc tga gca gcc ata ttg aca tcc t- $\left.3^{\prime}\right)$ was kindly provided by Angie Ribera (University of Colorado Health Sciences). The morpholino was injected into wildtype TL embryos at one- to two-cell stages at a concentration of $2 \mathrm{~mm}$. Pictures were taken using a 


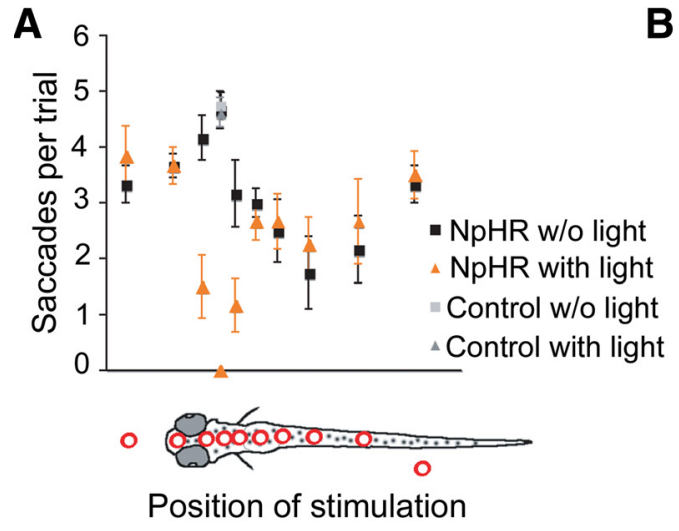

B

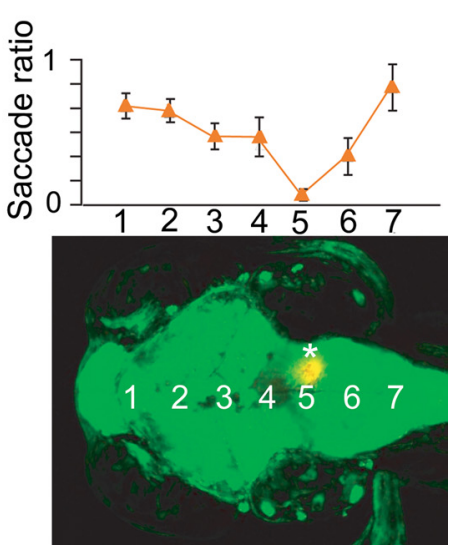

Figure 1. Optogenetic scanning with fiber optics identifies a hindbrain region necessary for saccade generation. $\boldsymbol{A}$, Coarse mapping of the brain using a 200- $\mu$ m-diameter optic fiber to locate regions in the brain that are important for horizontal eye movements during OKR. During trials, animals were continuously visually stimulated with a moving grating (conditions in all panels: contrast, $100 \%$; velocity, $10 \%$; spatial frequency, 0.05 cycles per degree), and the fiber light was alternately turned on and off for periods of $20 \mathrm{~s}$ each. The number of saccades during one trial was measured for either wildtype larvae (gray) or siblings carrying Et(E1b:Gal4-VP16)s1101t, Tg(UAS:NpHR-eYFP) s1987t (black and orange). The drawing in the bottom panel of $A$ shows the corresponding brain regions that were illuminated during the trial. A total of 158 trials were analyzed for the mapping experiments and five animals were used (three NpHR expressors, two nonexpressing siblings). Error bars represent SEM. B, Detailed mapping of the saccade phenotype along the midline in the brain. A 50- $\mu \mathrm{m}$-diameter optic fiber was used. The saccade ratio (number of saccades during NpHR activation divided by number of saccades without NpHR activation) approached 0 for position number 5 . Positions are shown in a z-projected confocal stack of a Et(E1b:Gal4-VP16)s1101t, Tg(UAS:NpHR-eYFP)s1987t, Tg(UAS: Kaede)s $1999 t$ carrying larvae. The asterisk indicates a photoconverted position corresponding to the experiment in Figure $2 B$.

Leica MZFLIII dissecting microscope connected to a Zeiss Axiocam MRc camera.

Expression analysis by in situ RNA hybridization of the scnllab gene was performed as previously described (Smear et al., 2007). An isoformspecific region within the scnllab gene was chosen as previously described (Novak et al., 2006).

Site-directed mutagenesis. A plasmid containing the wildtype $R n S c n 2 a$ gene positioned between the $5^{\prime}$ and $3^{\prime}$ noncoding regions of the Xenopus $\beta$-globin gene downstream of a T7 RNA polymerase promoter and upstream of a poly-A tail region was a kind gift from Alan L. Goldin (University of California, Irvine, Irvine, CA). The codon change recreating the $d i d y^{5552}$ mutation was introduced into the $R n S c n 2 a$ construct with a single PCR-digest step. The BglII and BlpI restriction sites were used to cut out $\sim 500 \mathrm{bp}$ of the RnScn $2 a$ region, containing the site of interest. The mutation was introduced into the RnScn $2 a$ gene using PCR with the following oligonucleotides: $R n S c n 2 a F W\left(5^{\prime}\right.$-acc gct gga aga tct gaa taa caa g-3'); and RnScn $2 a R V$ - (didy $\left.{ }^{5552}\right)\left(5^{\prime}\right.$-cac cac tgc tga gca gaa tcc taa- $\left.3^{\prime}\right)$. The PCR product was digested with BglII and BlpI restriction enzymes and subsequently ligated into the digested construct. Sequencing was performed to confirm the presence of the mutation in the RnScn2a construct and to ensure that no additional mutations were introduced during the cloning procedures.

Two-electrode voltage-clamp recordings from Xenopus oocytes. Capped cRNA was transcribed in vitro from a NotI linearized plasmid with the T7 mMessage mMachine kit (Ambion). The quality of the cRNA was confirmed by electrophoresis, and quantity was determined by photospectrometry (OD260). Stage V oocytes were removed from adult female Xenopus laevis frogs, prepared as previously described (Goldin, 1991). Approximately $20 \mathrm{ng}$ of cRNA was injected into Xenopus oocytes using a microinjector. Oocytes were incubated for $>48 \mathrm{~h}$ at $16^{\circ} \mathrm{C}$ in ND96 medium. Sodium currents were recorded by using a two-electrode voltageclamp set up in ND96 medium in the absence or presence of $400 \mathrm{~nm}$ tetrodotoxin (TTX). Capacitive transients and leak currents were eliminated by subtraction of currents remaining after TTX treatment. The voltage dependency of the sodium currents was determined by performing a voltage-injection protocol with steps between -95 and $+50 \mathrm{mV}$, with a holding potential of $-100 \mathrm{mV}$ and increments of $5 \mathrm{mV}$. Data analysis was performed using pClamp 8.0 software (Molecular Devices). Peak currents were analyzed and plotted against their voltages.

\section{Results}

\section{A small hindbrain region in} rhombomere 5 is necessary for saccade generation

To localize the neural circuitry responsible for saccade initiation in the larval zebrafish brain, we used an optogenetic stimulation approach (Fig. 1). Fish larvae ( $5 \mathrm{dpf}$ ) were mounted in agarose, placed inside a custom-built surround videotheater, and assayed for their eye movements in response to visual motion. We used the broadly expressing Et(E1b:Gal4VP16)s1101t transgenic line (Scott et al., 2007; Scott and Baier, 2009) to drive the expression of NpHR-mCherry in almost all neurons of the zebrafish CNS (Arrenberg et al., 2009). An optic fiber was coupled to a laser $(633 \mathrm{~nm})$ and positioned close to the surface of the brain. This configuration allowed us to simultaneously stimulate small brain areas and monitor eye movements while the animal was exposed to the visual stimulus presented on the surround video screens.

We first used a $200 \mu \mathrm{m}$ fiber (diameter) to coarsely map the brain and to control for the effect of scattered light reaching the retina (Fig. $1 A$ ). Finer mapping was then performed with a $50 \mu \mathrm{m}$ fiber (Fig. $1 B$ ). In most regions of the brain and spinal cord, OKR saccades were unaltered or only mildly affected by optical stimulation. In particular, illumination of the pretectum and tectum had no effect (Fig. 1A,B; supplemental Movie 1, available at www.jneurosci.org as supplemental material). Only at one position in the hindbrain, a small region in rhombomere 5 between the ears and near the midline, was our protocol successful in blocking OKR-associated saccades (Figs. $1 A, B, 2 B, C$; supplemental Movie 2, available at www.jneurosci.org as supplemental material). This position is distinct from the swiminducing neurons in the caudal hindbrain discovered by us previously (Arrenberg et al., 2009). Light stimulation of strictly the right side of this region resulted in inhibition of saccades to the right, while saccades to the left were not affected, and vice versa (Figs. $1 B, 2 B$; supplemental Movie 3, available at www.jneurosci. org as supplemental material). Slow eye movements were not blocked by inhibition of these cells either bilaterally or unilaterally (Fig. $2 B, C$ ).

\section{Optical stimulation of a small hindbrain area is sufficient for saccade generation}

We next used ChR2(H134R) to activate the neurons at the identified position. Light stimulation of ChR2-eYFP-expressing larvae was initially performed in the absence of visual stimulation. ChR2 activation elicited conjugate (binocularly coordinated) saccades in the ipsilateral and contralateral eye in the direction of the stimulated side (Fig. 2E, F; supplemental Movie 4, available at www.jneurosci.org as supplemental material). Optically induced saccades were on average less vigorous than naturally occurring saccades $\left(54.4 \pm 8.2\right.$ vs $\left.102.6 \pm 6.4^{\circ} / \mathrm{s}\right)$, but substantially faster than the fastest OKR slow phase measured $(10.5 \% \mathrm{~s})$. The latency between ChR2 activation and initial eye movement was $\sim 28 \mathrm{~ms}$. No other behavior was observed, such as tail movements or escapes, despite vicinity to the Mauthner cells 
in rhombomere 4. Illumination of other brain regions did not elicit rapid eye movements (Fig. 2D; supplemental Movie 5, available at www.jneurosci.org as supplemental material), with the exception of small areas rostral to the identified region (supplemental Movies 6 and 7, available at www.jneurosci.org as supplemental material). Stimulating these regions evoked short, jerky eye movements $\left(28.5 \pm 2.5^{\circ} / \mathrm{s}\right)$, which were restricted to the eye ipsilateral to the stimulation, in striking contrast to the bilateral saccades observed earlier.

To label the region responsible for saccade initiation, we used the photoconvertible fluorescent protein Kaede. Triple transgenic zebrafish larvae carrying Et(E1b: Gal4-VP16)s1101t, Tg(UAS:Kaede)s1999t, and either $\mathrm{Tg}$ (UAS:NpHR-mCherry)s1989t (Fig. $1 B)$ or $\mathrm{Tg}(\mathrm{UAS}$ :ChR2(H134R)eYFP)s1990t (Fig. 2G) were first optically stimulated to locate the saccade-generating region. The input to the optic fiber was then switched to a UV laser (365 nm), without altering its position, and the larva was illuminated with UV. We reported previously that this photoconversion protocol yields a very close estimate of the illuminated volume, because the difference in scattering between the two wavelengths is negligible (Arrenberg et al., 2009). The red-converted Kaede highlights the stimulated region in the zebrafish larval hindbrain, $\sim 20 \mu \mathrm{m}$ lateral from the midline (Fig. $2 G$ ). Imaging of this area with $40 \times$ magnification showed that a narrow column of $<100$ cells $(\sim 15$ in one optical plane) was converted by the UV light (Fig. 2H). Together, these data show that a small number of neurons in the larval hindbrain are necessary and sufficient to elicit a saccade, consistent with the existence of a burst generator in the larval fish brain.

\section{Optical stimulation of abducens motor} neurons in a specific enhancer-trap line does not produce saccades

We asked whether activation of abducens motor neurons alone could result in saccade-like eye movements. For this experiment, we used the enhancer trap line Et (hsp(600bp):Gal4-VP16)s1020t, which drives expression in Olig2-expressing neurons (Scott et al., 2007; Wyart et al., 2009). Olig2 is a transcription factor that marks the abducens motor neurons in zebrafish (Zannino and Appel, 2009). Photostimulation of ChR2 in the abducens motor neurons labeled by $\operatorname{Et}(h s p(600 b p)$ : Gal4-VP16)s1020t (Fig. 3A) did not evoke rapid eye movements, even when much greater light intensities were

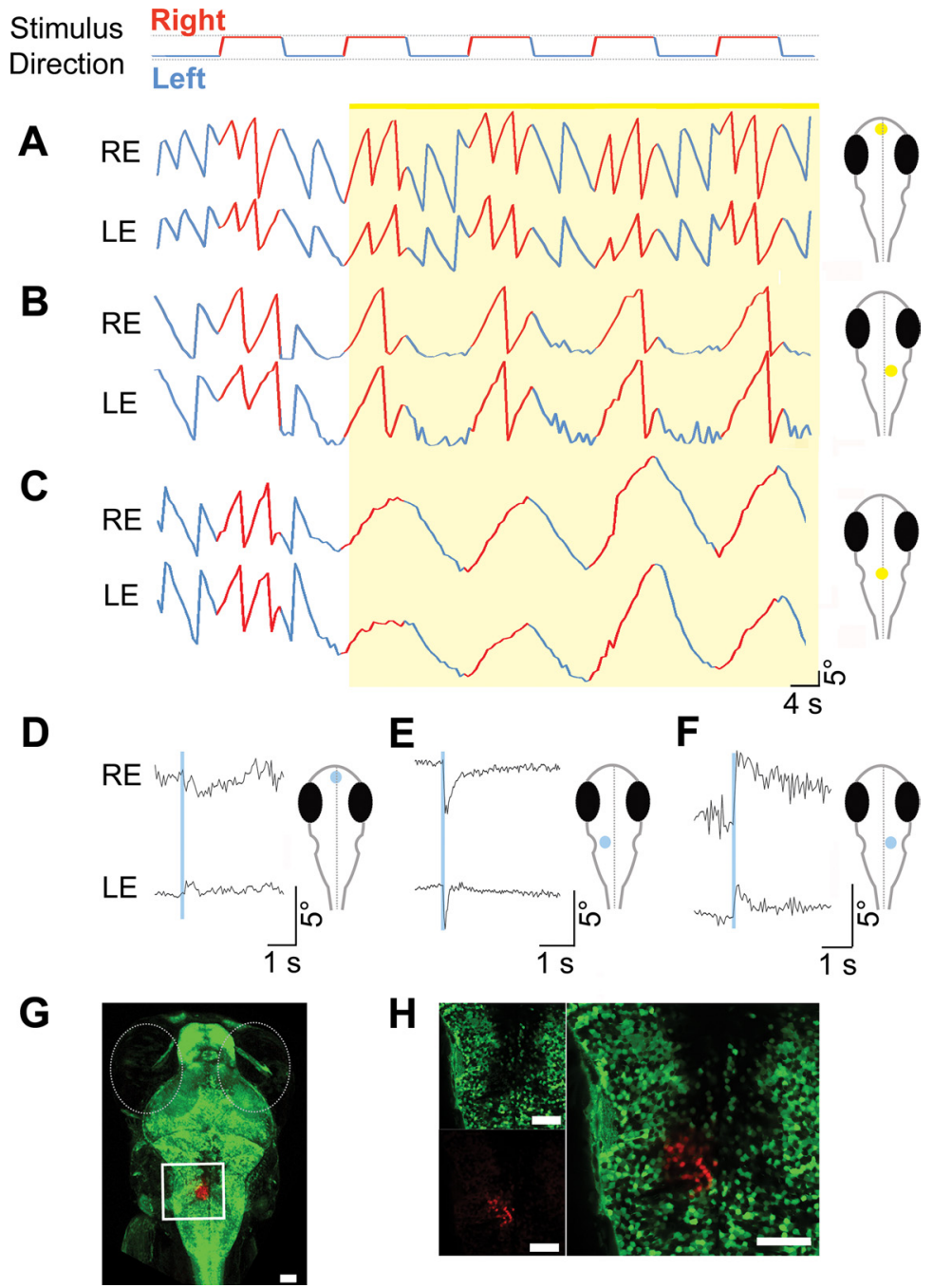

Figure 2. Optical manipulation of the hindbrain saccade-generating circuit with NpHR and ChR2. OKR stimulus conditions in panels $\mathrm{A}-\mathrm{C}$ were: contrast, $100 \%$; velocity, $\pm 10 \%$; spatial frequency, 0.05 cycles per degree. Larvae transgenic for $E t(E 1 b$ :Gal4VP16)s1101t, Tg(UAS:NpHR-eYFP)s1987t were visually stimulated with a direction-changing, drifting grating as indicated in the row above $\boldsymbol{A}$. $\boldsymbol{A}-\boldsymbol{C}$, Eye traces [right eye (RE) and left eye (LE)] during optogenetic stimulation of telencephalon (A), unilateral hindbrain $(\boldsymbol{B})$, and bilateral hindbrain $(\boldsymbol{C})$ are plotted. Yellow shading corresponds to the illumination of the brain region shown on the right next to the eye traces. Direction of the stimulus is color coded in the eye traces (red, right direction; blue, left direction). The location of stimulation in $\boldsymbol{B}$ is shown and photo converted in Figure $1 \boldsymbol{B}$, bottom (asterisk). $\boldsymbol{D}-\boldsymbol{F}$, Telencephalon (D) and lateral hindbrain $(\boldsymbol{E}, \boldsymbol{F})$ stimulation of Et(E1b:Gal4-VP16)s1101t, Tg(UAS:Kaede)s1999t, Tg(UAS:ChR2(H134R)-eYFP)s1990t larvae in the absence of a visual stimulus. Light pulses are shown in blue shading. Representative traces are shown (eight or more animals were tested in each condition). G, Low-magnification image ( $10 \times$ objective) of the expression pattern of Kaede in the animal tested in $\boldsymbol{D}-\boldsymbol{F}$. Kaede (unconverted, green) is expressed in most neurons. UV illumination with the same $50 \mu \mathrm{m}$ optic fiber at the identical location that is used for optical manipulation experiments in $E$ results in local photoconversion of Kaede (red) within a $50-\mu \mathrm{m}$ wide column. $\boldsymbol{H}$, Higher magnification ( $40 \times$ ) of converted area. Scale bars: $\boldsymbol{G}, \boldsymbol{H}, 50 \mu \mathrm{m}$.
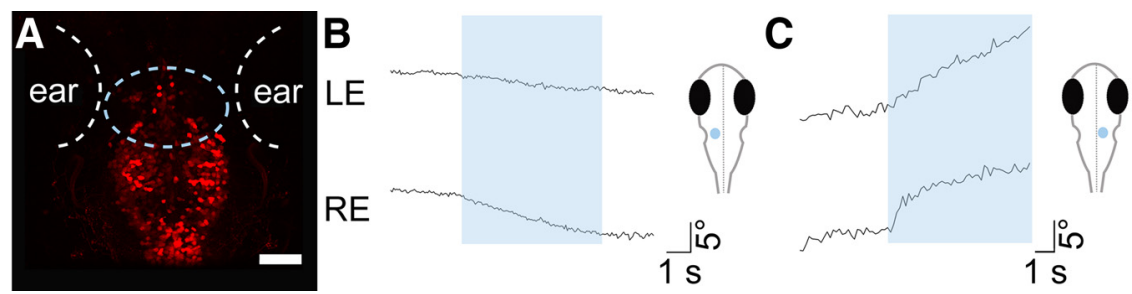

Figure 3. Optogenetic activation of the abducens motor neurons with ChR2 using a $50 \mu \mathrm{m}$ optic fiber. $A, Z$-projected confocal stack (40× objective) of the expression pattern of ChR2-mCherry in Et(hsp(600bp):Gal4-VP16)s1020t, Tg(UAS:ChR2(H134R)$m$ Cherry)s $1986 t$. The blue dashed oval between the ears indicates the region of photostimulation. Scale bar, $50 \mu \mathrm{m}$. B, C, Lateral hindbrain stimulation of Et(hsp(600bp):Gal4-VP16)s1020t, Tg(UAS:ChR2(H134R)-mCherry)s 1986t larvae. Light pulses are shown in blue shading. Blue dots between the ears indicate the region of photostimulation. More positive eye positions correspond to leftward eye movements in this figure, but to rightward eye movements in all other figures. 

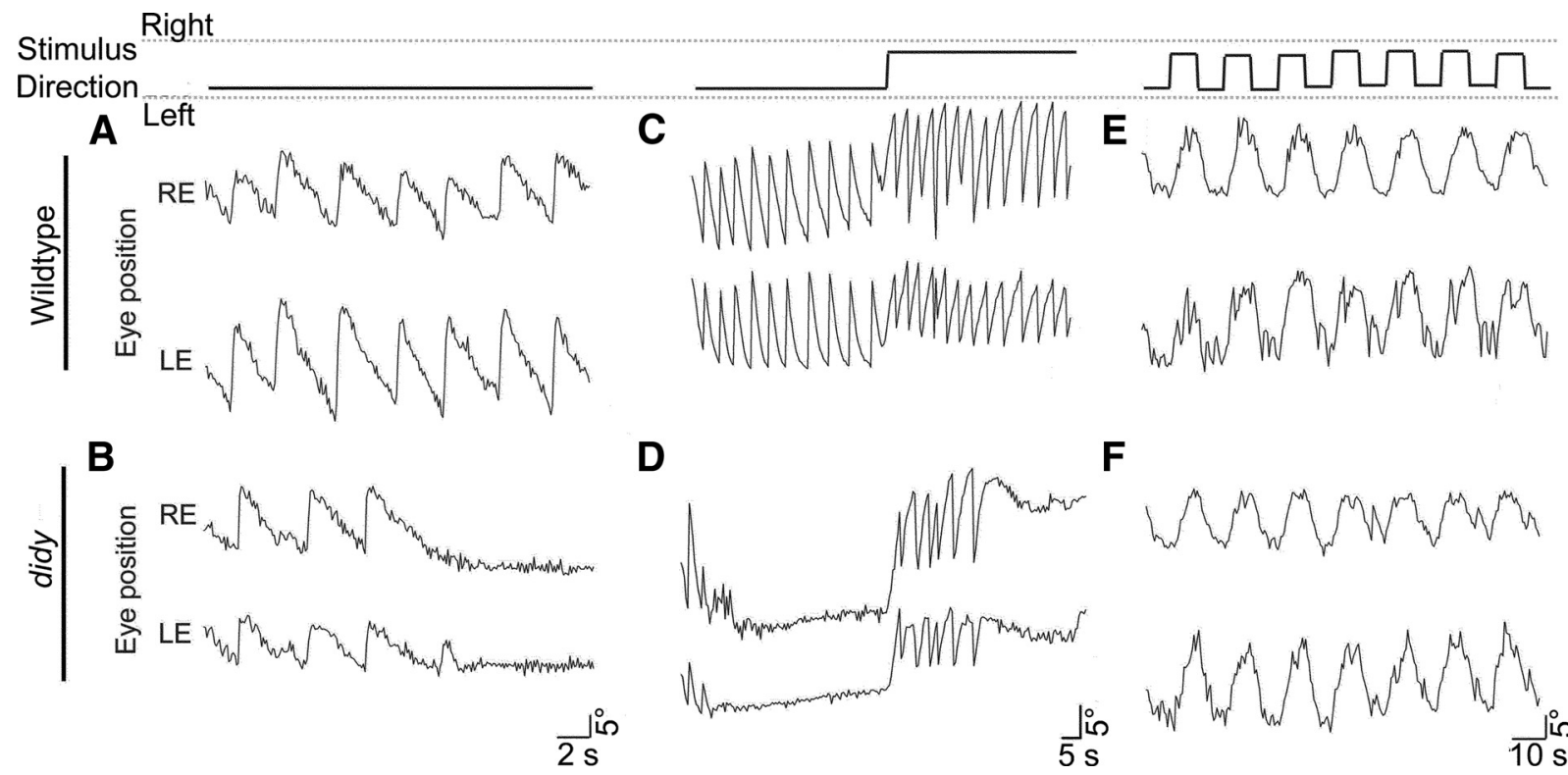

D

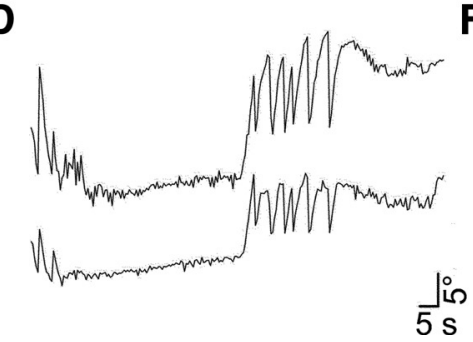

$\mathbf{F}$
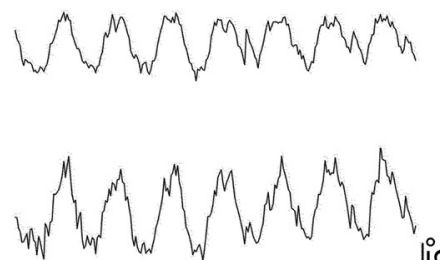

Figure 4. Optokinetic responses reveal a saccade generation defect in didy ${ }^{552}$ mutants ( $5 \mathrm{dpf}$ ). Stimulus conditions: contrast, $100 \%$; velocity. $\pm 10 \%$; spatial frequency; $0.05 \mathrm{cycles}$ per degree. Stimulus directions for $\boldsymbol{A}-\boldsymbol{F}$ are indicated in the top row of the figure. $\boldsymbol{A}, \boldsymbol{B}$, Representative traces of the horizontal position of the left eye (LE) and right eye (RE) of a wildtype and a didy larva in response to a drifting grating with a single direction, plotted as a function of time. Mutant larvae respond initially like wildtype larvae but then cease to generate saccades. $\boldsymbol{C}, \boldsymbol{D}$, Representative eye movement traces in response to a drifting grating with a single reversal of stimulus direction. $\boldsymbol{E}, \boldsymbol{F}$, Representative eye movement traces in response to an oscillating motion stimulus. Stimulus direction was changed every $8 \mathrm{~s}$. No difference is seen between wildtype and mutant larvae.
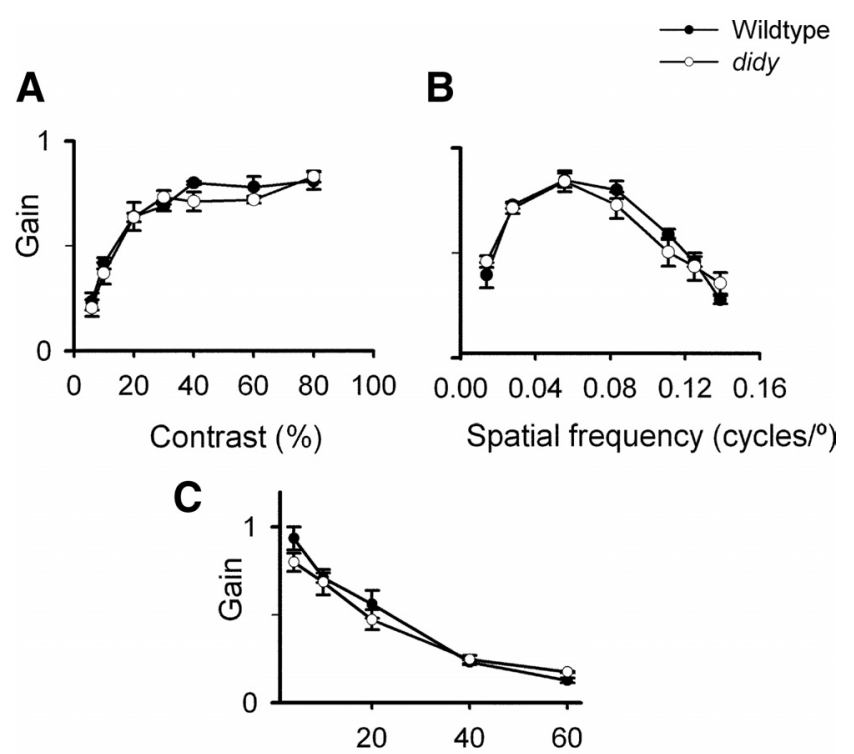

Stimulus velocity $(\% \mathrm{sec})$

Figure 5. Normal visual acuity and contrast sensitivity in didy mutants. Fish larvae ( $n=8$ for each genotype) were tested at $5 \mathrm{dpf}$. Stimulus conditions in all panels were (unless otherwise stated): contrast, $100 \%$; velocity, $10 \%$; spatial frequency, 0.05 cycles per degree. $A$, The slowphase velocity gain (OKR gain) of wildtype and didy mutants as a function of stimulus contrast. $B$, OKR gain of wildtype and didy mutants as a function of spatial frequency. $C$, OKR gain of wildtype and didy mutants as a function of stimulus velocity.

tested. Instead, activation of these neurons resulted in eye movements that were slower and more variable (Fig. $3 B, C$ ) than those seen in the broadly expressing Et(E1b:Gal4VP16)s1101t line (Fig. 2E,F). This result indicated that the saccade-generating neurons identified in rhombomere 5 of the $E t(E 1 b: G a l 4-V P 16) s 1101 t$ line are different from, and likely upstream of, the abducens motor neurons.
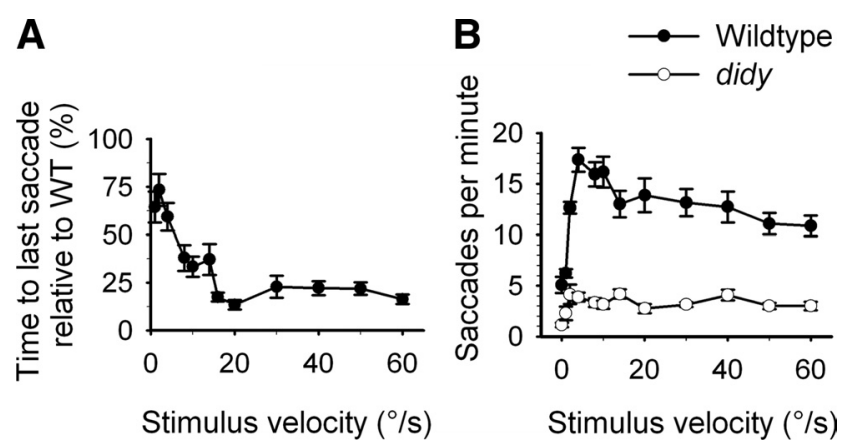

Figure 6. Exhaustion of saccade production in didy ${ }^{552}$ mutants is dependent on the number of prior saccades, not on stimulus velocity. Stimulus conditions: contrast, $100 \%$; spatial frequency, 0.05 cycles per degree. $A$, Time of the last saccade event that occured during a 2 min exposure to stimulus drifting in one direction $(n=8)$. $\boldsymbol{B}$, Saccade rate of wildtype (solid circle) and didy mutant (open circle) during the $0 \mathrm{KR}$ to a stimulus drifting in one direction as a function of stimulus velocity $(n=10)$. Error bars represent SEM.

\section{didy mutants fail to sustain saccades during the OKR in a direction-specific manner}

Two alleles of the didy gene were discovered in a large-scale screen for ethylnitrosourea-induced mutations disrupting the OKR (Muto et al., 2005). Whereas wildtype larvae manifest full-blown OKR behavior in response to a unidirectional, moving, largefield grating for many minutes, $d i d y^{5552}$ mutants execute approximately four (three to six) initial saccades and then lock their eyes in the most extreme peripheral position at the end of a slow phase (Fig. $4 A, B$ ). When the motion stimulus is ended, the mutants' eyes slowly drift to the position that is parallel to the body axis. When the direction of the motion stimulus is reversed, didy mutants perform a slow phase to the opposite side and subsequently perform another series of about four saccades, before the eyes become arrested again in the most extreme "pursuit" position (Fig. 4C,D). The occurrence of another series of saccades upon direction reversal shows that the saccade exhaustion in didy mu- 
tants is specific to the direction tested, i.e., the eye movement defect does not transfer between stimulus directions. When the direction of the motion stimulus was reversed every $8 \mathrm{~s}$, the slow phase of the OKR was indistinguishable between wildtype and didy mutants (Fig. 4E,F). Thus, the oculomotor system is not generally weakened, and the mutants are well capable of detecting the motion stimulus.

The didy mutation does not affect vision, saccade ballistics, or eye muscles

The OKR is evoked by visual stimulation, and any perturbation of its normal performance could be due to disruptions of its sensory inputs or motor outputs. To test for a visual defect, we determined the gain of the slow phase of didy mutant and wildtype larval zebrafish as a function of contrast, spatial frequency, and velocity of the stimulus. We observed no differences in sensitivity or acuity between wildtype and didy mutants over the range of stimuli tested (Fig. 5A-C). These data suggest that the didy ${ }^{552}$ mutation does not diminish visual perception.

Since slow phase and quick phase of the OKR employ the same set of motor neurons and muscles, and the slow phase is unaltered in the mutant, it seemed likely that the $d i d y^{5552}$ mutation does not affect the last stages of motor output. To further verify this assumption, we measured the ballistics of the saccades in response to a grating moving in a single direction. We found that saccades (if they occur) were very similar between wildtype and didy mutants in both amplitude and speed $(102.6 \% \pm 6.4$ SEM for wildtype; $110.1 \% \pm 6.4$ SEM for $d i d y^{5552}$ ), indicating that motor neuron discharges and eye muscles are intact in the mutant.

The saccade generation defect does not depend on stimulus velocity and recovers over time

We asked whether the ability to generate saccades in didy mutants was dependent on time of exposure to a moving grating (i.e., subsided after a defined time independent of stimulus velocity) or the number of preceding saccades (i.e., subsided after a defined number of saccades). We distinguished between these possibilities by varying the stimulus velocity over almost two orders of magnitude ( 1 to $60 \%$ s) while holding the spatial frequency of the grating constant. At each stimulus velocity, didy mutants performed about four saccades (Fig. 6B), demonstrating that the ability to sustain saccades was not dependent on time. Responding to faster stimulus velocities, the mutant performed the same number of saccades as for slow velocities, but in a shorter time (Fig. 6A).

The mutant was also less likely than the wildtype to initiate spontaneous saccades during free viewing when faced with a stationary grating (velocity 0 ). The rate dropped from approximately five saccades per minute in the wildtype to less than one in the mutant (Fig. $6 B$ ).

Finally, we tested the time scales on which the didy mutants recovered from their saccade-exhausted state. Two OKR trials (stimulus velocity, $+10 \%$ ) were performed in which the interval

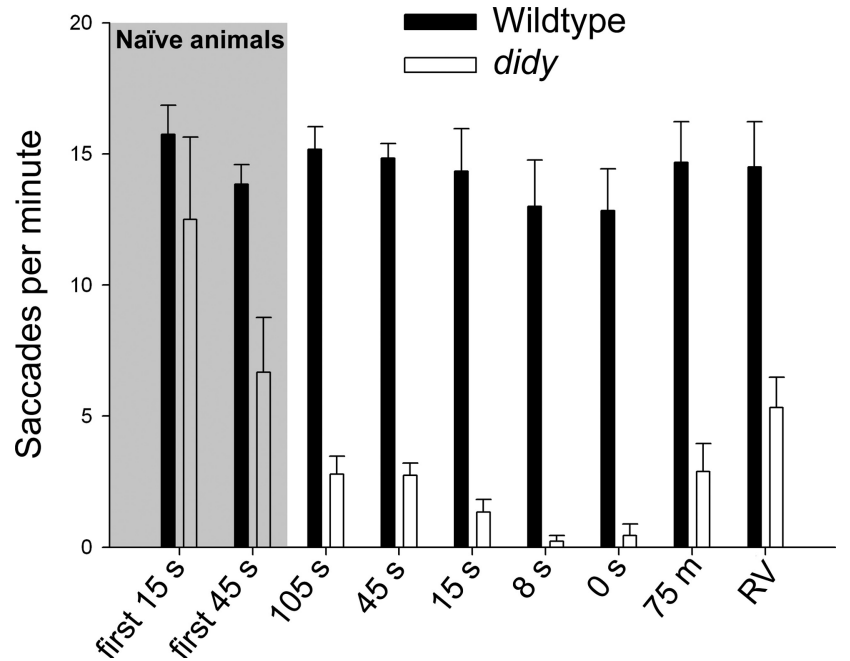

Figure 7. Recovery kinetics of saccade generation in didy mutants. Wildtype (solid bars) and didy mutants (open bars) were exposed twice, for $45 \mathrm{~s}$ each, to a stimulus drifting in one direction (velocity, $10 \% \mathrm{~s}$ ). The number of saccades during the second exposure was counted ( $n \geq 6$ animals for each genotype and condition). Time intervals (velocity, $0 \%$ ) between exposures to the motion stimulus were varied. The leftmost four bars (gray background) correspond to saccade rate during first exposure (naive animals). Following bars show saccade rates during the second 45 s trial performed after the indicated interval. RV stands for a reversal of the direction of the OKR stimulus (interval, 0 s). Error bars represent SEM.
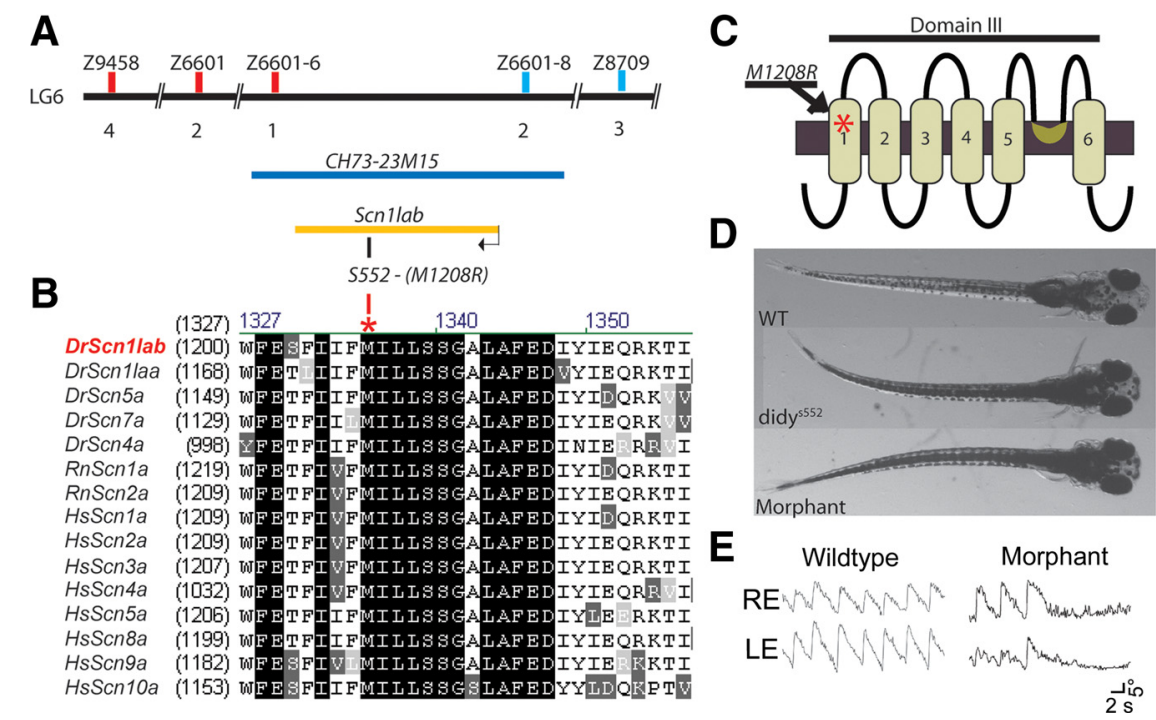

Figure 8. Molecular identification of didy as scn 1lab. A, Positional cloning of the didy gene. The didy ${ }^{552}$ mutation was mapped between markers z6601-6 and z6601-8, flanking the scn1lab gene. Both markers are located on BAC clone CH73-23M15. $B$, Alignment of the amino acid sequence neighboring the M1208R mutation identified in the didy ${ }^{552}$ allele to homologs from other vertebrate species. Dr, Danio rerio (zebrafish); Rn, Rattus norvegicus; Hs, Homo sapiens. C, Predicted location of the M1208R in domain III of the voltage-gated sodium channel Scn1lab (Nav1.1Lb). D, E, Morpholino knock down of scn1lab transcript in wildtype larvae resulted in a phenocopy of the didy ${ }^{552}$ mutation. $D$, Both mutant and morphant larvae are dark due to dispersed melanin pigment, indicating a neuroendocrine defect. $\boldsymbol{E}$, Unidirectional OKR of didy morphant larva shows a failure to sustain saccades similar to didy ${ }^{552}$ mutants.

between exposures to the moving grating was varied. During the interval, the stimulus grating was stationary (velocity 0 ). We observed that didy mutants regained their ability to generate saccades when intervals of $>45 \mathrm{~s}$ were used (Fig. 7). When the two exposures followed each other with an interval $<8 \mathrm{~s}$, the didy mutants were largely incapable of executing another saccade (Fig. 7). This finding suggests that the saccade-generating mechanism, once depleted, recovers on the order of tens of seconds in $d i d y^{552}$ mutants. 
A

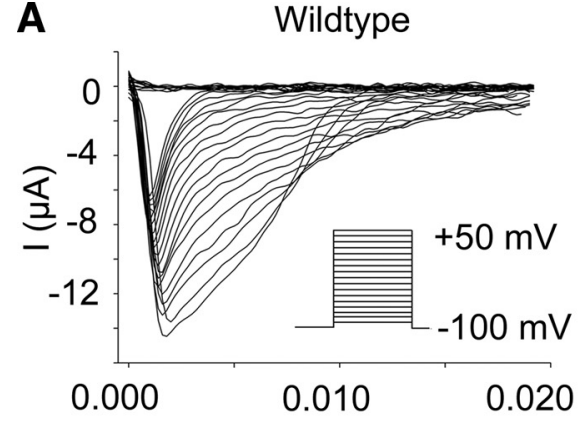

Time (s)

C

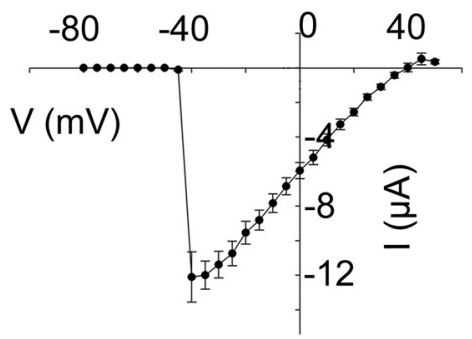

B

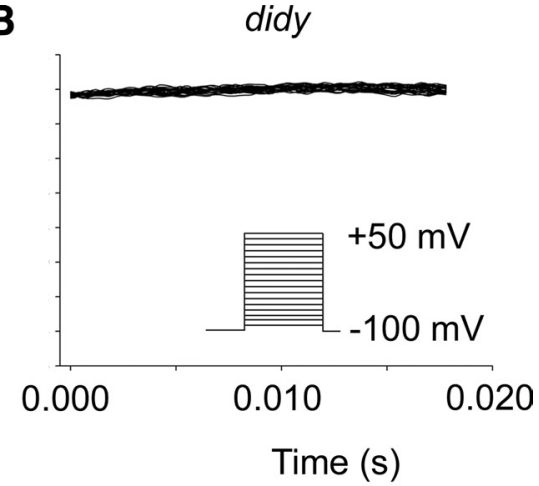

D

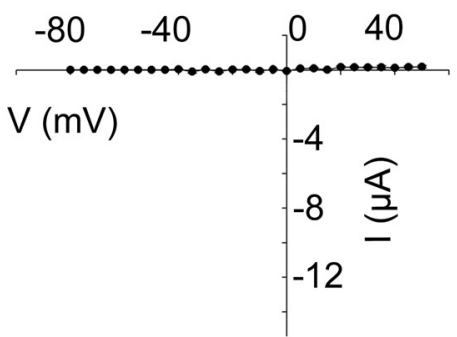

Figure 9. The mutant didy allele likely encodes a nonfunctional channel. Two-electrode voltage-clamp recordings were performed on Xenopus 0ocytes heterologously expressing rat Scn2a carrying the amino acid substitution equivalent to the didy ${ }^{552}$ mutation. Oocytes were voltage clamped with a holding potential of $-100 \mathrm{mV}$ followed by depolarizations in $5 \mathrm{mV}$ increments to $+10 \mathrm{mV} . \boldsymbol{A}, \boldsymbol{B}$, Representative traces of recordings from Xenopus oocytes transfected with either wildtype scn2a $(\boldsymbol{A})$ or mutant $\operatorname{scn} 2 a(\boldsymbol{B}) . \boldsymbol{C}, \boldsymbol{D}$, Voltage dependence of activation for wildtype $\operatorname{Sen} 2 \mathrm{a}(\boldsymbol{C}, n=12$, mean $\pm \operatorname{SEM})$ and mutant $\operatorname{Sen} 2 \mathrm{a}(\boldsymbol{D}, n=12$, mean \pm SEM).

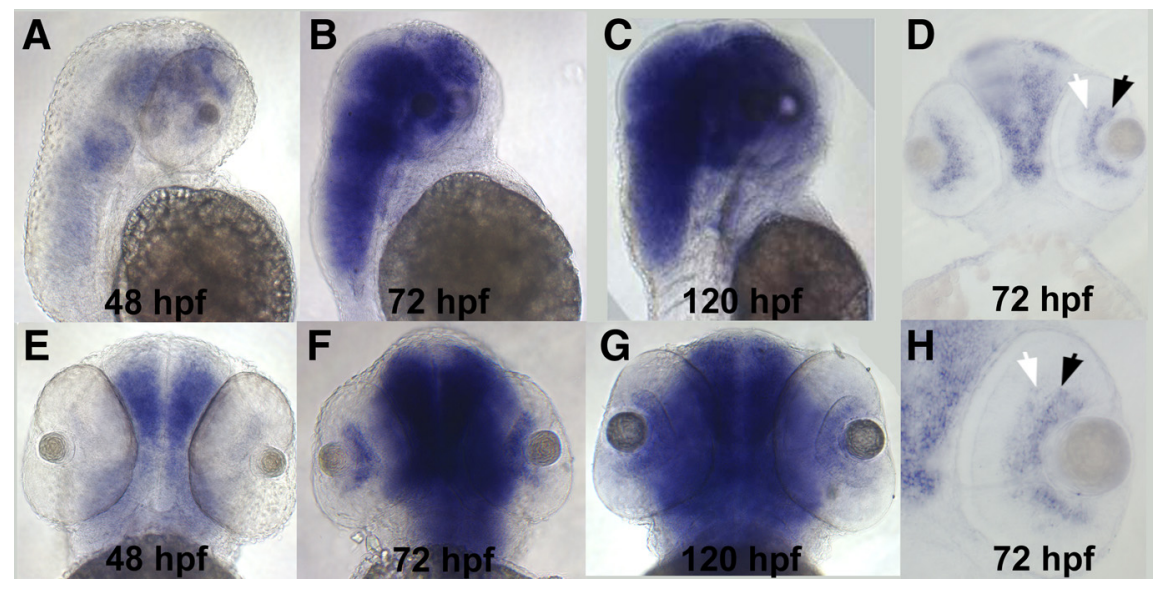

Figure 10. Expression pattern of scn 1lab in zebrafish larvae revealed by antisense digoxigenin-labeled RNA in situ hybridization. $\boldsymbol{A}, \boldsymbol{E}$, Lateral and ventral views, respectively, of whole-mount staining of $48 \mathrm{hpf}$ embryos. $\boldsymbol{B}, \boldsymbol{F}$, Lateral and ventral views, respectively, of whole-mount staining of $72 \mathrm{hpf}$ larvae. $C, G$, Lateral and ventral views, respectively, of whole-mount staining of $120 \mathrm{hpflarvae}$. $\boldsymbol{D}$, Vibratome section $(20 \mu \mathrm{m})$ showing a $72 \mathrm{hpflarval}$ retina and brain. $\boldsymbol{H}, 20 \times$ magnification of the eye. Most cells in the ganglion cell layer of the retina (black arrow), close to the lens, express scn1lab. Scattered cells in the inner nuclear layer (white arrow) are also scn1lab positive.

\section{Genetic and functional identification of $d i d y^{s 552}$ as a null allele of scn1lab}

To identify the mutated gene, we used a positional cloning approach. By means of a genome scan using 192 simple sequence repeat length polymorphism (SSLP) markers (Shimoda et al., 1999) distributed over the entire zebrafish genome, we mapped the $d i d y^{5552}$ mutation to a region on chromosome 6 between the markers $z 22712$ and $z 62065$. We scanned the genomic region between these two markers for additional simple sequence repeats and found two novel SSLPs, which we called z6601-6 and z6601-8 (see Materials and Methods). These markers flank the $d i d y^{552}$ mutation and are located on bacterial artificial chromosome (BAC) clone CH73-23M15 (Fig. $8 A$ ). Interrogation of the Ensembl database revealed that a single gene was located in this interval, scn1lab. Sequencing of the scn1lab open reading frame revealed a T-to-G mutation in the didy ${ }^{5552}$ cDNA, which results in an amino acid substitution from a methionine (M) to an arginine (R) at position 1208. The scn1lab gene encodes a voltage-gated sodium channel (Nav1.1lb), which consists of four domains of six transmembrane helices (Catterall, 2001). The M1208 residue is situated in the predicted first transmembrane helix of the third domain of this channel and is conserved across the family of paralogous genes in mice, rats, humans, and zebrafish (Fig. $8 B, C$ ).

To confirm the identity of the gene, we used a morpholino antisense approach to knock down scnllab in zebrafish larvae. The morpholino was targeted against the translation start site (ATG) of scnllab. The morphants showed the same external phenotype as the mutants (Fig. 8D). Moreover, morphant fish were impaired in saccade initiation similar to didy mutants (Fig. $8 E$ ). In summary, genetic linkage, DNA sequence, and morpholino phenocopy indicate that the didy ${ }^{5552}$ allele is a loss-of-function mutation of scnllab.

We next studied the effect of the didy $y^{552}$ mutation on the gating properties of this channel in Xenopus oocytes. Genes encoding voltage-gated sodium channels are notoriously difficult to clone due to toxicity in Escherichia coli. We therefore took advantage of the available $\operatorname{Scn} 2 \mathrm{a}$ clone from Rattus norvegicus ( $\mathrm{RnScn} 2 a$ ) (Kearney et al., 2001) and substituted the $\mathrm{M}$ with an $\mathrm{R}$ at the position equivalent to residue 1208 to generate a mutant channel. We injected wildtype and mutant Rnscn $2 a$ mRNA into Xenopus oocytes and performed two-electrode voltage-clamp recordings. Oocytes injected with wildtype $R n S c n 2 a$ showed voltage-gated sodium peak currents of $\sim 12 \mu \mathrm{A}$ at $-40 \mathrm{mV}$ (Fig. $9 A, C)$. Mutant channels, in contrast, were unable to carry currents at any voltage (Fig. $9 B, D$ ). These results corroborate the identity of the didy gene as scnllab and indicate that the $d i d y^{5552}$ mutation generates a complete loss-of-function allele.

The didy saccade deficit is reversed by activating ChR 2 in the putative saccade generator

Wholemount RNA in situ hybridization at 48, 72, and $120 \mathrm{~h}$ postfertilization (hpf) showed that transcript levels are qualitatively unchanged in didy $y^{5552}$ mutants, suggesting that the mutation does not affect mRNA stability. This was confirmed by 
quantitative PCR (data not shown). Similar to other voltage-gated sodium channels (Novak et al., 2006), the gene is broadly expressed in the brain (Fig. 10). The gene product is, however, not ubiquitous. In the retina, scnllab is restricted to retinal ganglion cells and a subset of amacrine cells (Fig. 10D,H). Consistent with the behavioral phenotype, the expression pattern of scnilab overlaps with the saccade-generating region identified above. However, its broad expression pattern is uninformative with regard to its site and mechanism of action in the didy mutant.

We hypothesized that depolarization of the saccade-generating neurons with ChR2 might restore saccades in didy mutants. For this experiment, scnllab morpholino was injected into Et(E1b:Gal4-VP16)s1101t and Tg(UAS:ChR2(H134R)-eYFP)s1990t embryos to generate larvae with a deficit in sustaining saccades. We exposed these larvae to a unidirectional OKR stimulus and waited for their saccades to run down. As before, didy morphants performed only approximately four saccades, but light stimulation at the position of the putative saccade generator rescued saccade initiation (Fig. 11D). Light injection elicited multiple conjugate saccades of normal speeds and amplitudes similar to those in wildtype animals, which were always directed to the stimulated side and precisely time locked to the light pulse (Fig. $11 B, D$ ). For wildtype animals, 16 of 18 light injections, and for didy morphants 13 of 15 light injections resulted in a saccade. Optical stimulation of other brain regions was ineffective (Fig. $11 \mathrm{~A}, \mathrm{C}$ ). For wildtype animals, 1 of 20 light injections, and for didy morphants 0 of 12 light injections resulted in a saccade. These experiments suggest that the downstream motor neurons or muscles are less, if at all, affected by the didy $y^{552}$ mutation and locate the defect within or upstream of the putative saccade generator.

\section{Discussion}

This work has discovered the location of hindbrain neurons in larval zebrafish that are necessary and sufficient for saccade generation. The properties of this circuitry (Fig.

12) suggest that it is homologous to the one inferred, using electrophysiology, in adult goldfish (Pastor et al., 1994; Aksay et al., 2001, 2007; Major et al., 2004) and, possibly, to the burst generator in mammals (Fuchs and Kaneko, 1981; Scudder et al., 2002). Optical stimulation of ChR2-expressing neurons on just one side of the hindbrain results in a saccade of both eyes in the direction of the stimulated side. This is in line with the connectivity reported in other vertebrates; the burst neurons activate motor neurons directly (abducens) and indirectly (oculomotor) to drive the ipsilateral lateral rectus muscle and the contralateral medial rectus muscle, thus en-

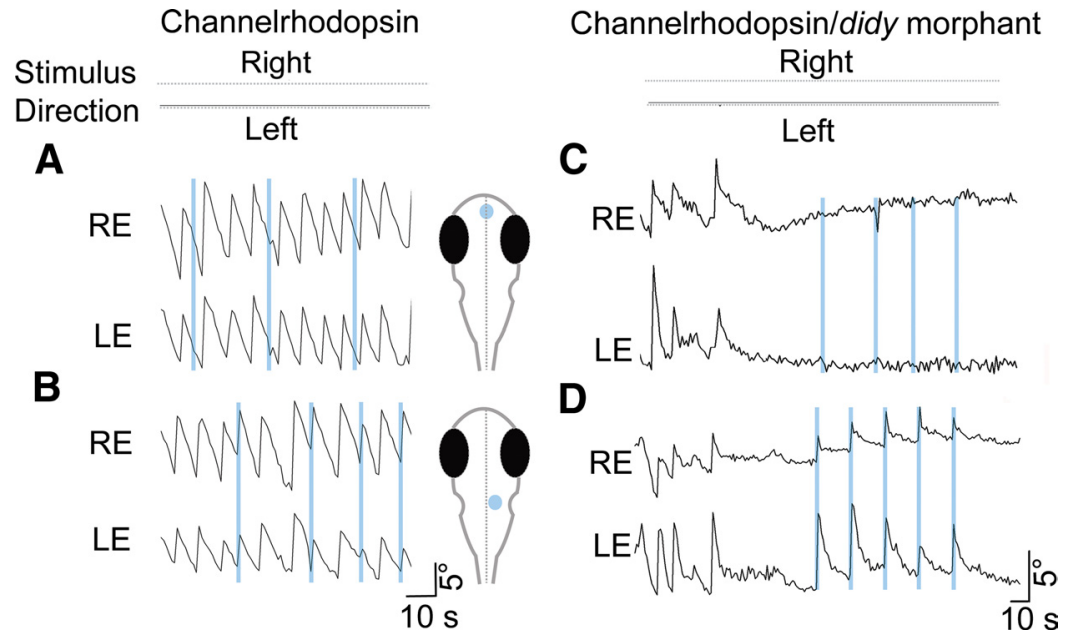

Figure 11. Restoration of saccades in didy $y^{552}$ mutants by optical stimulation of the putative saccade generator region with ChR2. $A, C$, Control: no extra saccades are evoked by photostimulation of a telencephalon region of wildtype larva $(\boldsymbol{A})$ or didy morphants (C) carrying Et(E1b:Gal4-VP16)s1101t, Tg(UAS:ChR2(H134R)-eYFP)s1990t during exposure to a drifting grating. B, D, Saccades are consistently triggered by stimulation of the rhombomere 5 region in the same animals. Representative traces are shown ( $n \geq 3$ animals for each condition).

\section{A Saccade generation in mammals}

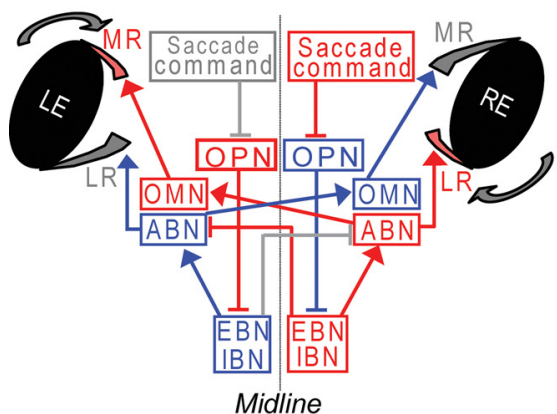

Red: Active before rightward saccade (arrow). Blue: Inhibited. Gray: Inactive or unknown.

Figure 12. Schematic drawing of circuitry responsible for horizontal saccade generation in mammals and teleosts. $A$, Saccadegenerating circuitry in primates. Neurons in the midbrain inhibit the OPNs, directly or indirectly through activation of inhibitory interneurons. The inhibition of OPN results in short, rapid firing of EBNs, which monosynaptically activate motor neurons in the abducens nucleus (ABN). These motor neurons activate the lateral rectus muscle (LR) on the ipsilateral side. Abducens internuclear neurons in the $A B N$ project to the contralateral oculomotor nucleus (OMN) to activate oculomotor motor neurons. These motor neurons activate the medial rectus muscle (MR) of the contralateral eye. Simultaneously, IBNs inhibit the contralateral ABN, which results in a relaxation of the ipsilateral MR and the contralateral LR. B, Putative framework of the circuitry for horizontal saccade generation in zebrafish larvae. Neurons in the midbrain generate a saccade command that directly or indirectly activates the hindbrain saccade generator on only one side of the animal (right side is shown). The saccade generator activates motor neurons and internuclear neurons in the ipsilateral ABN. The ABN motor neurons activate the LR on the ipsilateral side. The ABN internuclear enrons project to the contralateral $\mathrm{OMN}$ to activate oculomotor motor neurons. The oculomotor motor neurons activate the MR of the eye contralateral to the activated saccade generator. Thus, a saccade generated in the right side of the hindbrain manifests as rightward eye rotation of both eyes. The circuitry upstream of the saccade generator, especially the existence of neurons homologous to the OPN, has not been demonstrated. abling the production of conjugate eye movements (Fig. 12A). Our data suggest that the quick phase of the OKR in larval zebrafish is generated by a circuit that is comparable in organization to the saccadic burst generator engaged during free viewing in primates (Fig. $12 \mathrm{~B})$. This conservation is remarkable, considering the divergent functions of the two behaviors, the phylogenetic distance of zebrafish from mammals, the absence of a fovea in zebrafish, and the well documented differences in the neural pathways that underlie the initiation of these two types of eye movements (superior colliculus/optic tectum in free viewing and nucleus of the optic tract/pre- 
tectum in the OKR) (Ilg and Hoffmann, 1991, 1996; Masseck and Hoffmann, 2009).

From a forward genetic screen, which used the OKR as a behavioral screening assay (Muto et al., 2005), we isolated a zebrafish mutant in NaV1.1lb, which is encoded by the scn1lab gene. Heterologous expression and electrophysiology in Xenopus oocytes showed that the mutation likely generated a null allele of this voltage-gated sodium channel. Perhaps surprisingly, this "channelopathy" results in a selective defect in the sustenance of saccadic eye movements. Several brain functions tested appear normal, including visual perception and ocular pursuit, as well as swimming scoots and escape responses. However, the mutants fail to inflate their swim bladder, have a deficit in the neural control of pigmentation, and do not survive beyond $14 \mathrm{dpf}$, indicating that closer analysis will reveal additional neurological defects. Several mutations in genes encoding human Nav1 proteins have been identified and have been shown to result in epilepsy syndromes (Kearney et al., 2001; Goldin, 2003; Ogiwara et al., 2007; Ragsdale, 2008). The zebrafish didy mutant may provide an opportunity to study disease aspects that are inaccessible in mammalian models, such as long-term changes in synaptic function or cell-type-specific effects. For zebrafish neuroscience, the $d i d y^{5552}$ mutant is interesting because it is one of very few mutations isolated so far that disrupt integrative functions of the brain, while sparing both the sensory surface and the motor output. Most other "behavioral" mutants identified in forward genetic screens affect neural development, sensory processing, or simple locomotor behaviors (Brockerhoff et al., 1995; Granato et al., 1996; Muto et al., 2005).

Nav1.1lb belongs to a family of at least eight voltage-gated sodium channels encoded in the vertebrate genome (Novak et al., 2006). Although its mutation causes a rather specific behavioral defect, we found that this channel is expressed rather broadly in the CNS. This counterintuitive result is best explained by partial redundancy with other Nav1 channels, which are coexpressed in most neurons and can compensate for the absence of one of the channels. We hypothesize that the specificity of the didy phenotype arises primarily from functional compensation of voltagegated sodium channels in most other neurons outside the saccade generator circuit. Homeostatic regulation of membrane excitability, e.g., downregulation of voltage-gated potassium channels, may further ameliorate the phenotype.

To begin to understand the molecular causes of the behavioral phenotype, we considered known properties of this protein family. Voltage-gated sodium channels exist in three states: "closed" (activatable); "open"; and "inactive." Transitions from one state to another are largely unidirectional, from closed to open, from open to inactive, and from inactive to closed. Neurons become unable to generate action potentials when a large fraction of channels are in the inactive state, e.g., following sustained depolarization or repetitive firing. Importantly, the inactive state comprises at least two distinct conformations with characteristic recovery times, a fast reversible form with recovery kinetics in the millisecond range and a slowly reversible form (Rudy, 1978; Quandt, 1988; Jung et al., 1997; Mickus et al., 1999). The slowly reversible form may be unavailable for activation for seconds or tens of seconds due to a structural rearrangement of the channel protein (Jung et al., 1997; Toib et al., 1998; Carr et al., 2003). Deletion of one of the channels, as in didy mutants, is expected to reduce channel density. Prolonged stimulation could deplete the pool of activatable channels and render the neurons unexcitable. This gradual loss of excitability could underlie the mutants' inability to sustain saccades. Intriguingly, our saccade recovery ex- periments showed that didy mutants needed between 8 and $45 \mathrm{~s}$ of recovery to be able to perform saccades again, a time that is in agreement with the recovery time of sodium channels in the slowly reversible inactive state. Optical stimulation of ChR2expressing neurons in the hindbrain restored saccades. We propose that the $\mathrm{Na}^{+}$currents added by activated ChR2 depolarized the affected neurons and thus helped them surpass the firing threshold; the remaining endogenous voltage-gated sodium channels were then sufficient to carry the ensuing spikes.

Several lines of evidence suggest that the $d i d y^{5552}$ mutation is selective to premotor circuitry. First, in the context of the OKR, the defect appears limited to saccade generation; photoreception, motion detection, and slow-phase eye movements are all intact. Second, photoactivation of the putative saccade generator triggers saccades, even in didy mutants whose ability to produce saccades had previously been exhausted, suggesting that motor neurons and eye muscles are unaffected. Third, ChR2 stimulation of abducens motor neurons in the Et(hsp (600bp):Gal4VP16)s1020t line did not elicit saccades, indicating that cells upstream of the motor neurons were triggering the saccades observed in the broadly expressing Et(E1b:Gal4-VP16)s1101t line. Fourth, the saccade generation defect transfers between eyes, but is direction specific; after exhaustion of saccades in one direction, reversal of the OKR stimulus still elicits saccades in the opposite direction, as one would expect from the connectivity of the burst neurons as shown in our model (Fig. 12). Fifth, assuming evolutionary conservation of the circuit, the deficit is unlikely to be presynaptic to EBNs, because OPN input is inhibitory (Fig. 12); an inability to spike in OPN neurons should therefore result in an increased number of saccades, not in fewer saccades. (For the same reason, we expect optical activation of OPNs to repress saccades and optical silencing of these neurons to facilitate saccades.) Based on these five arguments, we favor the burst generator as the site of didy action. Alternatively or in addition, a defect of circuit elements inhibiting the OPNs (if they exist in teleosts), such as the dorsal long-lead burst neurons described previously (Kamogawa et al., 1996), could also contribute to the didy phenotype.

In conclusion, this work has identified a region in the zebrafish larval hindbrain whose activity is necessary and sufficient for saccades to occur. The underlying circuit appears to be homologous, or at least functionally equivalent, to the mammalian burst generator. A newly discovered "channelopathy" perturbs this circuitry, rendering it unable to sustain action potentials over long periods of time. Behavioral experiments involving optical activation have allowed us to infer the basic layout of the saccadegenerating circuitry in larval zebrafish, including its bilateral connections and lateralized directional specificity. Finally and more generally, we have shown here that ChR2 stimulation can be used to mimic neurological functions, several synapses removed from the sensory or motor peripheries. Optogenetic tools can thus complement more conventional approaches to studying circuit function in the zebrafish brain.

\section{References}

Aksay E, Baker R, Seung HS, Tank DW (2000) Anatomy and discharge properties of pre-motor neurons in the goldfish medulla that have eyeposition signals during fixations. J Neurophysiol 84:1035-1049.

Aksay E, Gamkrelidze G, Seung HS, Baker R, Tank DW (2001) In vivo intracellular recording and perturbation of persistent activity in a neural integrator. Nat Neurosci 4:184-193.

Aksay E, Olasagasti I, Mensh BD, Baker R, Goldman MS, Tank DW (2007) Functional dissection of circuitry in a neural integrator. Nat Neurosci 10:494-504. 
Arrenberg AB, Del Bene F, Baier H (2009) Optical control of zebrafish behavior with halorhodopsin. Proc Natl Acad Sci U S A 106:17968-17973.

Beck JC, Gilland E, Tank DW, Baker R (2004) Quantifying the Ontogeny of Optokinetic and Vestibuloocular Behaviors in Zebrafish, Medaka, and Goldfish. J Neurophysiol 92:3546-3561.

Beck JC, Rothnie P, Straka H, Wearne SL, Baker R (2006) Precerebellar Hindbrain Neurons Encoding Eye Velocity During Vestibular and Optokinetic Behavior in the Goldfish. J Neurophysiol 96:1370-1382.

Brainard DH (1997) The Psychophysics Toolbox. Spatial Vision 10:433-436.

Brockerhoff SE, Hurley JB, Janssen-Bienhold U, Neuhauss SC, Driever W, Dowling JE (1995) A behavioral screen for isolating zebrafish mutants with visual system defects. Proc Natl Acad Sci U S A 92:10545-10549.

Carr DB, Day M, Cantrell AR, Held J, Scheuer T, Catterall WA, Surmeier DJ (2003) Transmitter modulation of slow, activity-dependent alterations in sodium channel availability endows neurons with a novel form of cellular plasticity. Neuron 39:793-806.

Catterall WA (2001) Structural biology: a 3D view of sodium channels. Nature 409:988-989, 991.

Easter SS Jr, Nicola GN (1996) The development of vision in the Zebrafish (Danio rerio). Dev Biol 180:646-663.

Fuchs AF, Kaneko CRS (1981) A brain stem generator for saccadic eye movements. Trends Neurosci 4:283-286.

Glimcher PW (2003) The neurobiology of visual-saccadic decision making. Annu Rev Neurosci 26:133-179.

Goldin AL (1991) Expression of ion channels by injection of mRNA into Xenopus oocytes. Methods Cell Biol 36:487-509.

Goldin AL (2003) Mechanisms of sodium channel inactivation. Curr Opin Neurobiol 13:284-290.

Granato M, van Eeden FJ, Schach U, Trowe T, Brand M, Furutani-Seiki M, Haffter P, Hammerschmidt M, Heisenberg CP, Jiang YJ, Kane DA, Kelsh RN, Mullins MC, Odenthal J, Nüsslein-Volhard C (1996) Genes controlling and mediating locomotion behavior of the zebrafish embryo and larva. Development 123:399-413.

Ilg UJ, Hoffmann KP (1991) Responses of monkey nucleus of the optic tract neurons during pursuit and fixation. Neurosci Res 12:101-110.

Ilg UJ, Hoffmann KP (1996) Responses of neurons of the nucleus of the optic tract and the dorsal terminal nucleus of the accessory optic tract in the awake monkey. Eur J Neurosci 8:92-105.

Jung HY, Mickus T, Spruston N (1997) Prolonged sodium channel inactivation contributes to dendritic action potential attenuation in hippocampal pyramidal neurons. J Neurosci 17:6639-6646.

Kamogawa H, Ohki Y, Shimazu H, Suzuki I, Yamashita M (1996) Inhibitory input to pause neurons from pontine burst neuron area in the cat. Neurosci Lett 203:163-166.

Kanda T, Iwamoto Y, Yoshida K, Shimazu H (2007) Glycinergic inputs cause the pause of pontine omnipause neurons during saccades. Neurosci Lett 413:16-20.

Kearney JA, Plummer NW, Smith MR, Kapur J, Cummins TR, Waxman SG, Goldin AL, Meisler MH (2001) A gain-of-function mutation in the sodium channel gene Scn2a results in seizures and behavioral abnormalities. Neuroscience 102:307-317.

Lopez-Barneo J, Darlot C, Berthoz A, Baker R (1982) Neuronal activity in prepositus nucleus correlated with eye movement in the alert cat. J Neurophysiol 47:329-352.

Major G, Baker R, Aksay E, Mensh B, Seung HS, Tank DW (2004) Plasticity and tuning by visual feedback of the stability of a neural integrator. Proc Natl Acad Sci U S A 101:7739-7744.

Marsh E, Baker R (1997) Normal and adapted visuooculomotor reflexes in goldfish. J Neurophysiol 77:1099-1118.

Masseck OA, Hoffmann KP (2009) Comparative neurobiology of the optokinetic reflex. Ann N Y Acad Sci 1164:430-439.

Mickus T, Jung HY, Spruston N (1999) Slow sodium channel inactivation in CA1 pyramidal cells. Ann N Y Acad Sci 868:97-101.

Muto A, Orger MB, Wehman AM, Smear MC, Kay JN, Page-McCaw PS,
Gahtan E, Xiao T, Nevin LM, Gosse NJ, Staub W, Finger-Baier K, Baier H (2005) Forward genetic analysis of visual behavior in zebrafish. PLoS Genet 1:e66.

Neuhauss SC, Biehlmaier O, Seeliger MW, Das T, Kohler K, Harris WA, Baier H (1999) Genetic disorders of vision revealed by a behavioral screen of 400 essential loci in zebrafish. J Neurosci 19:8603-8615.

Novak AE, Taylor AD, Pineda RH, Lasda EL, Wright MA, Ribera AB (2006) Embryonic and larval expression of zebrafish voltage-gated sodium channel $\alpha$-subunit genes. Dev Dyn 235:1962-1973.

Ogiwara I, Miyamoto H, Morita N, Atapour N, Mazaki E, Inoue I, Takeuchi T, Itohara S, Yanagawa Y, Obata K, Furuichi T, Hensch TK, Yamakawa K (2007) Nav1.1 localizes to axons of parvalbumin-positive inhibitory interneurons: A circuit basis for epileptic seizures in mice carrying an scnla gene mutation. J Neurosci 27:5903-5914.

Pastor AM, De la Cruz RR, Baker R (1994) Eye position and eye velocity integrators reside in separate brainstem nuclei. Proc Natl Acad Sci U S A 91:807-811.

Pelli DG (1997) The VideoToolbox software for visual psychophysics: transforming numbers into movies. Spat Vis 10:437-442.

Quandt FN (1988) Modification of slow inactivation of single sodium channels by phenytoin in neuroblastoma cells. Mol Pharmacol 34:557-565.

Ragsdale DS (2008) How do mutant Navl.1 sodium channels cause epilepsy? Brain Res Rev 58:149-159.

Rudy B (1978) Slow inactivation of the sodium conductance in squid giant axons. Pronase resistance. J Physiol 283:1-21.

Scott EK, Baier H (2009) The cellular architecture of the larval zebrafish tectum, as revealed by Gal4 enhancer trap lines. Front Neural Circuits $3: 13$.

Scott EK, Mason L, Arrenberg AB, Ziv L, Gosse NJ, Xiao T, Chi NC, Asakawa K, Kawakami K, Baier H (2007) Targeting neural circuitry in zebrafish using GAL4 enhancer trapping. Nat Methods 4:323-326.

Scudder CA, Kaneko CS, Fuchs AF (2002) The brainstem burst generator for saccadic eye movements; a modern synthesis. Exp Brain Res 142: $439-462$.

Shimoda N, Knapik EW, Ziniti J, Sim C, Yamada E, Kaplan S, Jackson D, de Sauvage F, Jacob H, Fishman MC (1999) Zebrafish genetic map with 2000 microsatellite markers. Genomics 58:219-232.

Smear MC, Tao HW, Staub W, Orger MB, Gosse NJ, Liu Y, Takahashi K, Poo MM, Baier H (2007) Vesicular glutamate transport at a central synapse limits the acuity of visual perception in zebrafish. Neuron 53:65-77.

Straka H, Beck JC, Pastor AM, Baker R (2006) Morphology and physiology of the cerebellar vestibulolateral lobe pathways linked to oculomotor function in the goldfish. J Neurophysiol 96:1963-1980.

Strassman A, Highstein SM, McCrea RA (1986a) Anatomy and physiology of saccadic burst neurons in the alert squirrel monkey. I. Excitatory burst neurons. J Comp Neurol 249:337-357.

Strassman A, Highstein SM, McCrea RA (1986b) Anatomy and physiology of saccadic burst neurons in the alert squirrel monkey. II. Inhibitory burst neurons. J Comp Neurol 249:358-380.

Toib A, Lyakhov V, Marom S (1998) Interaction between duration of activity and time course of recovery from slow inactivation in mammalian brain $\mathrm{Na}^{+}$channels. J Neurosci 18:1893-1903.

Tomlinson RD, Robinson DA (1984) Signals in vestibular nucleus mediating vertical eye movements in the monkey. J Neurophysiol 51:1121-1136.

Wyart C, Del Bene F, Warp E, Scott EK, Trauner D, Baier H, Isacoff EY (2009) Optogenetic dissection of a behavioural module in the vertebrate spinal cord. Nature 461:407-410.

Yoshida K, McCrea R, Berthoz A, Vidal PP (1982) Morphological and physiological characteristics of inhibitory burst neurons controlling horizontal rapid eye movements in the alert cat. J Neurophysiol 48:761-784.

Zannino DA, Appel B (2009) Olig $^{2+}$ precursors produce abducens motor neurons and oligodendrocytes in the zebrafish hindbrain. J Neurosci 29: 2322-2333. 